\title{
Pulmonary hypertensive vascular changes in lungs of patients with sudden unexpected death. Emphasis on congenital heart disease, Eisenmenger syndrome, postoperative deaths and death during pregnancy and postpartum
}

\author{
Dimitra Krexi, ${ }^{1}$ Mary N Sheppard ${ }^{2}$
}

${ }^{1}$ Medical School, Aristotle University of Thessaloniki, Thessaloniki, Greece ${ }^{2}$ CRY Centre of Cardiovascular Pathology, Cardiovascular Sciences Research Centre, St George's University of London, London, UK

\section{Correspondence to} Professor Mary N Sheppard, CRY Centre of Cardiovascular Pathology, Cardiovascular Sciences Research Centre, St George's University of London, Cranmer Terrace, London SW17 ORE, UK; m.sheppard@sgul.ac.uk

Received 15 August 2014 Revised 23 September 2014 Accepted 30 September 2014 Published Online First 23 October 2014

\section{CrossMark}

To cite: Krexi D, Sheppard MN. J Clin Pathol 2015;68:18-21.

\begin{abstract}
Aims Pulmonary hypertension (PH) in asymptomatic patients is a rare cause of sudden death. This study aims to determine the incidence of this entity and raise awareness among pathologists.

Methods We retrospectively investigated 44 cases of sudden unexpected death in relation to PH in patients not on antihypertensive therapy. This is the largest pathological study reported.

Results We report 44 cases of sudden death due to $\mathrm{PH}$ in which 28 (63.63\%) were female and $16(36.36 \%)$ were male, and the age range was from 5 days to 93 years old (mean age: $24 \pm 20$ ). The majority had no clinical evidence of $\mathrm{PH}$ prior to death with none on therapy. The majority died at rest, 18 cases $(40.90 \%)$, while 7 patients $(15.90 \%)$ died following cardiac surgery and 7 patients (15.90\%) during pregnancy or postpartum, 6 of whom had congenital heart disease. The cause of PH was recognised as congenital heart disease in 27 patients $(61.36 \%)$, 14 of whom had simple congenital heart disease, such as atrial or ventricular septal defect, and 13 had complex congenital heart disease with associated atrial septal defect or ventricular septal defect. The remaining 17 patients (29.55\%) suffered from primary PH due to plexiform arteriopathy, veno-occlusive disease and thromboembolic disease. Extensive sampling of the lungs is required to detect the lesions microscopically in these conditions. Conclusions It is important that clinicians and pathologists be aware of the risk of sudden unexpected death in asymptomatic patients with $\mathrm{PH}$, especially in those with congenital heart disease, after cardiac surgery or pregnancy.
\end{abstract}

\section{INTRODUCTION}

Pulmonary hypertension $(\mathrm{PH})$ is diagnosed when pulmonary artery pressure is above $25 \mathrm{~mm} \mathrm{Hg}$ at rest and $30 \mathrm{~mm} \mathrm{Hg}$ during exercise. It is a devastating, life-threatening disorder with no curative options, characterised by elevated pulmonary vascular resistance and secondary right ventricular failure. The aetiologies of pulmonary arterial hypertension (PAH) are multiple and its pathogenesis is complex. ${ }^{1} \mathrm{PH}$ can develop in association with many different diseases and its presence is nearly always associated with reduced survival. The prognosis and management of $\mathrm{PH}$ is largely dependent upon its underlying aetiology and severity of disease. It can be primary or secondary. ${ }^{2}$
Regardless of aetiology, the final common pathway of $\mathrm{PH}$ is right heart failure and death. The average survival after diagnosis is $<4$ years. New therapy has led to increased survival. In a recent study, $\mathrm{PH}$ was the direct cause of death (right heart failure or sudden death) in $44 \%$ of patients; $\mathrm{PH}$ contributed to, but did not directly cause, death in $37(44 \%)$ patients; and the death was not related to $\mathrm{PH}$ in the remaining cases $(n=7 ; 8.3 \%)$. All these patients had been symptomatic and on treatment for their $\mathrm{PH} .{ }^{3}$ Sudden death is very rare in patients with no prior symptoms, and a careful histological examination of the lungs is necessary to confirm the diagnosis. There have been previous reports describing sudden unexpected death from undiagnosed $\mathrm{PH}^{4-6}$

We present 44 patients who died suddenly and had PH at autopsy without previous treatment. This is the largest pathological study reported. These cases raise awareness of $\mathrm{PH}$ as a cause of sudden unexpected death and the importance of lung histology at autopsy in these circumstances. ${ }^{7}$

\section{METHODS}

The Cardiac Risk in the Young (CRY) Cardiovascular pathology unit at St George's Medical School, London, acts as a specialist tertiary cardiac pathology centre for sudden cardiac death (SCD) in the UK. We defined SCD as an unexpected death occurring within $24 \mathrm{~h}$ of onset of symptoms. We found 44 cases diagnosed with $\mathrm{PH}$. The diagnosis of $\mathrm{PH}$ was made in all these cases due to histological changes of widespread and severe $\mathrm{PH}$ in pulmonary arteries with medial hypertrophy, intimal fibrosis and occlusion with plexiform lesions. These were considered severe enough to be linked to the death. In those with cardiac conditions at autopsy, there were no new cardiac findings at autopsy to explain the sudden death which is presumed to be due to a cardiac arrhythmia in most cases. The patient's age, sex, circumstances of death and past medical history were obtained from the referring pathologist/coroner. All patients underwent toxicology screen which was negative in all 44 cases. The data of the postmortem clinical and histopathological findings in each case were collected prospectively. Pathological analysis of all lungs was performed by MNS with the consent of the coroner and the next of kin, according to the specific guidlines. $^{5}$ 


\section{Statistical analysis}

Characteristics of the population were described as means, SDs $( \pm S D)$ for continuous variables and counts with presentations for all variables.

\section{RESULTS}

\section{Patients' characteristics}

Forty-four cases of sudden death due to $\mathrm{PH}$ from the database, from which 28 (63.63\%) were female and 16 (36.36\%) were male. The average age was $24 \pm 20$, and the age range was from 5 days to 93 years old. All had been well prior to death with none on therapy for $\mathrm{PH}$.

\section{Circumstances of death}

The circumstances of death reported from the referring coroner include the following: majority died at rest, 18/44 (40.90\%). Death occurred unexpectedly postoperatively in 7 patients, all following cardiac surgery (15.90\%), who had been well with no cardiac dysfunction or arrhythmias, and during pregnancy or postpartum in 7 patients, 6 of whom had congenital heart disease (CHD) (15.90\%). Circumstances were unknown in 12 patients $(27.27 \%)$.

\section{Autopsy findings}

All had right ventricular hypertrophy at autopsy with enlarged pulmonary artery branches. Those with CHD had appropriate cardiac findings with or without surgery with no surgical complications found in those who had recent surgery. In all these congenital cardiac cases, the lungs were macroscopically normal. In those with primary $\mathrm{PH}$ with or without associated conditions, the lungs were macroscopically normal, and usually 2 sections from each lung lobe were taken at autopsy with $\mathrm{H} \& \mathrm{E}$ staining, as well as Elastin van Gieson, to visualise the blood vessels. In arterio-venous malformation case, a large malformation was noted on the surface of the left lower lobe of the lung with several underlying dilated channels around it. In thromboemboli disease, there were obstructive lesions in the smaller lobar vessels filled with pale firm tissue in all lobes with focal scars on the pleural surface of the lungs in keeping with old infarcts. There were no fresh thrombi in the pulmonary arterial branches.

\section{Causes of $\mathrm{PH}$}

\section{Congenital heart disease}

The most common cause of $\mathrm{PH}$ in our study was CHD ( $n=27$ or $61.36 \%)$, with the majority $(n=22$ or $81.48 \%)$ being $\leq 35$ years old, and the age range from 5 days to 56 years old. Simple CHD was identified in 14 patients (51.85\%), four had atrial septal defect, four ventricular septal defect, one aortic atresia with ventricular septal defect, four patent ductus arteriosus and one atrioventricular septal defect. In 7 cases, the patients had correction of the defect; in 7 , no surgery was done due to diagnosis of Eisenmengers (Intracardiac shunt that reverses to a right-to-left shunt due to irreversible $\mathrm{PH}$ ). Complex CHD with associated atrial septal defect or ventricular septal defect was seen in 13 patients (48.15\%). All these had surgery in the past, with 3 having Eisenmenger's syndrome. All these cases had plexiform lesions in the lungs associated with intimal obstruction and medial hypertrophy of the blood vessels at autopsy (figure 1A). The surgery for their CHD had no complications at autopsy which would have explained their sudden unexpected death.
Primary $\mathrm{PH}$ and those with associated causes

There were 17 cases $(29.55 \%)$ of primary $\mathrm{PH}$ due to plexiform arteriopathy (7), (one of whom was pregnant) veno-occlusive disease (6) (figure 1B) and thromboembolic disease (1), (figure 1C) antithrombin-3 deficiency associated with scleroderma (1), pulmonary arterio-venous malformation (1) and giant cell vasculitis of pulmonary arteries with no vasculitis elsewhere (1) (figure 1D).

\section{DISCUSSION}

This study highlights PH detected in the lung at autopsy in cases of sudden unexpected death. This is an important finding as this will make a valuable contribution in explaining the sudden death. The pathologist must be aware of occult $\mathrm{PH}$ as a contributing cause of sudden unexpected death in a variety of age groups ${ }^{6}$ and especially in patients with CHD. Cardiac arrhythmias are important contributors to morbidity and mortality in patients with $\mathrm{PH}^{8}$ The only way to make the diagnosis rests with a carefully performed autopsy and histological sampling of the lungs. Right ventricular hypertrophy and dilatation will usually be the first clue at autopsy. Careful scrutiny of many histological sections, usually 2 from each lobe of lung with particular attention to vascular structures is necessary. Plexiform lesions may be obscured by pulmonary oedema and congestion and will be missed if there is only a cursory review of the material. Vascular changes can be notoriously heterogeneous and variable throughout the lung in $\mathrm{PH}^{9}{ }^{9}$ One needs to look carefully especially in the smaller arterioles and preacinar arterioles for the classic evidence of medial hypertrophy, intimal thickening, blocked vessels and outgrowth of plexiform vein-like lesions around the blocked vessels with or without fibrinoid necrosis in the blocked vessel walls. Significant medial wall abnormalities are also present in the pulmonary trunk including fibrosis, atypical elastic pattern, cystic medial degeneration and atheroma. ${ }^{10}$

Pulmonary vascular disease in adults with $\mathrm{CHD}$ and especially its extreme expression, the Eisenmenger syndrome, can lead to sudden death, ${ }^{11}$ often in adulthood. ${ }^{12}$ Arrhythmias are frequent late sequelae in patients with Eisenmenger physiology. In the series reported by Daliento et al, ${ }^{12 a} 42 \%$ of patients with Eisenmenger had supraventricular arrhythmias on routine ECG or $24 \mathrm{~h}$ Holter monitoring during long-term follow-up. ${ }^{13}$ In patients who experienced sudden death, no symptoms of heart failure, history of arrhythmias or features of haemodynamic abnormalities were observed in $15 \%$ of cases. ${ }^{14}$

These arrhythmias combined with the congenital abnormalities in the heart make a sudden arrhythmic death likely. The pathologist must be aware that despite surgery for CHD, vascular changes in the lung persist and progress over the years leading to right ventricular hypertrophy and dilatation which will make a significant contribution to the death. The heart at autopsy will show no surgical complications and it is essential to take histological samples of the lungs. Adult cardiology is faced with a new challenging patient population. Since only a few congenital heart defects can be cured, regular follow-up during adult life is of major importance. Despite optimal treatment, the patients will develop long-term complications, such as arrhythmias, sudden death, $\mathrm{PH}$ and heart failure. Acute complications, such as fatal arrhythmias, aortic dissection or rupture, endocarditis, cerebral events due to embolism, bleeding or abscesses, and pulmonary embolism or bleeding must be looked for an autopsy. ${ }^{15}$ Deaths following surgery in CHD include low output failure as most common, but PH and SCD are also prominent. ${ }^{16}$ 


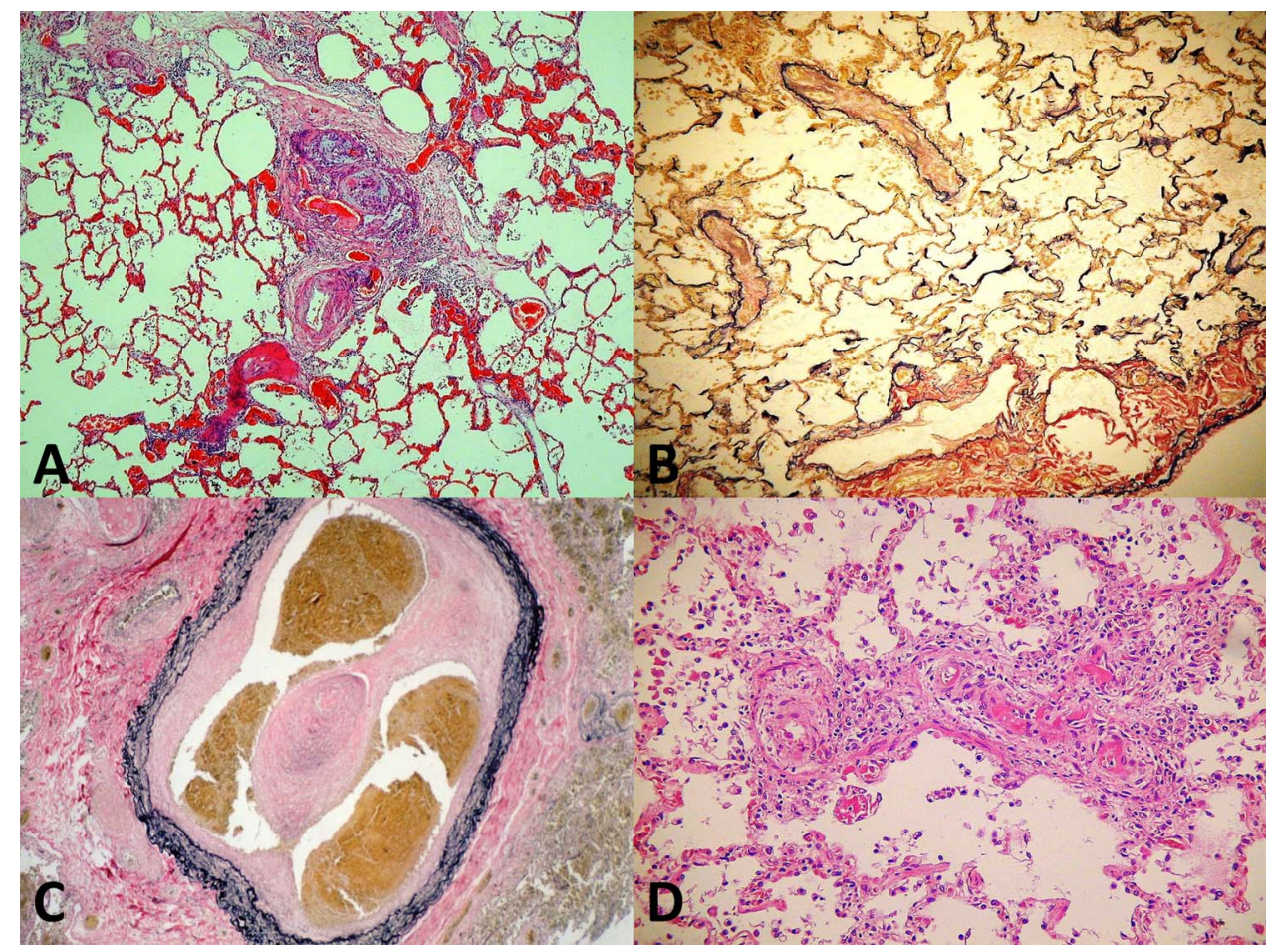

Figure 1 (A) shows a plexiform lesion in the lung associated with intimal obstruction and medial hypertrophy of arteriole, with fibrinoid necrosis of vessel wall. $H \& E \times 200$. (B) Primary pulmonary hypertension due to veno-occlusive disease in the lung. Note veins with narrow lumen due to fibrosis. Elastin van Gieson $\times 200$. (C) Pulmonary hypertension due to thromboembolic disease with recanalised pulmonary artery. Elastin van Gieson $\times 600$. (D) Pulmonary hypertension due to antithrombin-3 deficiency, with fibrin thrombi in small alveolar vessels within lung with intimal proliferation and narrowing. $\mathrm{H} \& \mathrm{E} \times 400$.

This study also emphasises death in pregnancy and following delivery with PH. Pregnancy with $\mathrm{CHD}$ and $\mathrm{PH}$ is associated with a markedly increased risk of adverse cardiovascular events and death for delivery and in the postpartum period. ${ }^{17-20} \mathrm{PAH}$, including Eisenmenger syndrome, has a risk of mortality in pregnancy of $10 \%-40 \%{ }^{21}$ Again pathologists dealing with maternal deaths need to be aware of this and sample the lungs. Primary pulmonary hypertension (PPH) may be a cause of sudden unexpected death with few, if any, earlier signs or symptoms, and once the autopsy diagnosis of PPH is established the pathologist must be aware that the entity may be familiar, and the family physician and surviving relatives should be informed. ${ }^{4}$ In these cases, the direct cause of death may be the vascular changes in the lungs combined with right ventricular hypertrophy and dilatation with terminal cardiac arrhythmia. The histological changes in veno-occlusive disease, chronic thromboembolic disease, antiphospholipid syndrome, hypertension associated with arterio-venous malformation and vasculitis are well described and the pathologist needs to be aware of these changes when the lungs are being examined. A trichrome stain is vital to highlight vascular changes in both arteries and veins. ${ }^{22}$

Left main coronary artery compression syndrome, pulmonary artery dissection, pulmonary artery rupture and severe haemoptysis are reported as complications leading to sudden death encountered more often in patients with PH. ${ }^{23}$ We had none of these complications in our series.

This study highlights the value of a full autopsy with histology in sudden unexpected death as in children ${ }^{25}$; the autopsy can be valuable in adults with and without $\mathrm{CHD}$, postcardiac surgery and in maternal deaths. The message is, always take lung histology, which may explain the sudden death when cardiac congenital lesions are stable with superadded right-sided cardiac hypertrophy and dilatation.

\section{Take home messages}

- Pulmonary hypertension is common in congenital heart disease and may contribute to sudden unexpected death. Patients may be asymptomatic clinically prior to death.

- Always take lung histology. In primary and secondary pulmonary hypertension, the lungs may be macroscopically normal.

- Right ventricular hypertrophy and dilation point at autopsy to pulmonary hypertension.

- The risk of sudden unexpected death in asymptomatic patients with pulmonary hypertension is higher in those with congenital heart disease, after cardiac surgery or pregnancy.

Contributors MNS: conceived the review question, developed the protocol and performed part of writing or editing of the protocol and review. MNS: made an intellectual contribution to the review, and approved the final version and review prior to submission. DK: conceived the review question, developed the protocol and review, coordinated the protocol and review development, and completed the first draft of the protocol and review. DK: made an intellectual contribution to the review, and approved the final version of the review prior to submission. MNS: conceived the statistical analysis protocol and reviewed all of the statistical analysis prior to submission. Both authors are guarantors of the review.

Funding UK based charity - Cardiac Risk in the Young (CRY). Grant number 63529. Competing interests None. 
Patient consent Obtained.

Provenance and peer review Not commissioned; externally peer reviewed.

\section{REFERENCES}

1 Guignabert C, Dorfmuller P. Pathology and pathobiology of pulmonary hypertension. Semin Respir Crit Care Med 2013;34:551-9.

2 McGlothlin D. Classification of pulmonary hypertension. Heart Fail Clin 2012; 8:301-17.

3 Tonelli AR, Arelli V, Minai OA, et al. Causes and circumstances of death in pulmonary arterial hypertension. Am J Respir Crit Care Med 2013;188:365-9.

4 Brown DL, Wetli CV, Davis JH. Sudden unexpected death from primary pulmonary hypertension. J Forensic Sci 1981;26:381-6.

5 Kawato $\mathrm{H}$, Hitosugi M, Kido M, et al. An autopsy case of sudden death in a boy with primary pulmonary hypertension: a case report. Med Sci Law 2005;45:361-3.

6 Srigley JA, Pollanen MS. Sudden death with clinically undiagnosed pulmonary hypertension. J Clin Forensic Med 2005;12:264-7.

7 Bolster MA, Hogan J, Bredin CP. Pulmonary vascular occlusive disease presenting as sudden death. Med Sci Law 1990;30:26-8.

8 Rajdev A, Garan H, Biviano A. Arrhythmias in pulmonary arterial hypertension. Prog Cardiovasc Dis 2012;55:180-6.

9 Tuder RM, Stacher E, Robinson J, et al. Pathology of pulmonary hypertension. Clin Chest Med 2013;34:639-50.

10 Prapa M, McCarthy KP, Dimopoulos K, et al. Histopathology of the great vessels in patients with pulmonary arterial hypertension in association with congenital heart disease: large pulmonary arteries matter too. Int J Cardiol 2013;168:2248-54.

11 Miyazaki A, Sakaguchi $H$, Ohuchi $H$, et al. The clinical characteristics of sudden cardiac arrest in asymptomatic patients with congenital heart disease. Heart Vessels Published Online First: 27 Nov 2013.

12 Greutmann M, Tobler D, Kovacs AH, et al. Increasing mortality burden among adults with complex congenital heart disease. Congenit Heart Dis Published Online First: 7 Jul 2014 12a Daliento L, Somerville J, Presbitero P, et al. Eisenmenger syndrome. Factors relating to deterioration and death. Eur Heart J 1998;19:1845-55.

13 Diller GP, Gatzoulis MA. Pulmonary vascular disease in adults with congenital heart disease. Circulation 2007:115:1039-50.

14 Koyak Z, Harris L, de Groot JR, et al. Sudden cardiac death in adult congenital heart disease. Circulation 2012:126:1944-54.

15 Baumgartner H, Dabritz S. [Congenital heart disease in adulthood]. Med Klin (Munich) 2008;103:135-42.

16 Ma M, Gauvreau K, Allan CK, et al. Causes of death after congenital heart surgery. Ann Thorac Surg 2007;83:1438-45.

17 Opotowsky AR, Siddiqi OK, D'Souza B, et al. Maternal cardiovascular events during childbirth among women with congenital heart disease. Heart 2012;98:145-51.

18 Bowater SE, Thorne SA. Management of pregnancy in women with acquired and congenital heart disease. Postgrad Med J 2010;86:100-5.

19 Curry R, Swan L, Steer PJ. Cardiac disease in pregnancy. Curr Opin Obstet Gynecol 2009;21:508-13.

20 Rosengarten D, Kramer MR. [Pregnancy in pulmonary arterial hypertension patients]. Harefuah 2013;152:547-51, 563,562.

21 Katsuragi S, Yamanaka K, Neki R, et al. Maternal outcome in pregnancy complicated with pulmonary arterial hypertension. Circ J 2012;76: 2249-54.

22 Lantuejoul S, Sheppard MN, Corrin B, et al. Pulmonary veno-occlusive disease and pulmonary capillary hemangiomatosis: a clinicopathologic study of 35 cases. AmJ SurgPathol 2006;30:850-7.

23 Demerouti EA, Manginas AN, Athanassopoulos GD, et al. Complications leading to sudden cardiac death in pulmonary arterial hypertension. Respir Care 2013;58:1246-54.

24 Correa Rde A, Silva LC, Rezende CJ, et al. Pulmonary hypertension and pulmonary artery dissection. J Bras Pneumol 2013;39:238-41.

25 Gatzoulis MA, Sheppard MN, Ho SY. Value and impact of necropsy in paediatric cardiology. Heart 1996;75:626-31. 